PROCEEDINGS OF THE

AMERICAN MATHEMATICAL SOCIETY

Volume 140, Number 2, February 2012, Pages 731-739

S 0002-9939(2011)11301-7

Article electronically published on September 21, 201

\title{
SMOOTH LIE GROUP ACTIONS ARE PARAMETRIZED DIFFEOLOGICAL SUBGROUPS
}

\author{
PATRICK IGLESIAS-ZEMMOUR AND YAEL KARSHON \\ (Communicated by Chuu-Lian Terng)
}

\begin{abstract}
We show that every effective smooth action of a Lie group $G$ on a manifold $M$ is a diffeomorphism from $G$ onto its image in $\operatorname{Diff}(M)$, where the image is equipped with the subset diffeology of the functional diffeology.
\end{abstract}

\section{INTRODUCTION}

The group Diff $(M)$ of diffeomorphisms of a manifold $M$, and subgroups that preserve certain geometric structures (for example, the group of symplectomorphisms of a symplectic manifold) are important objects of research. Such groups are often considered heuristically as infinite dimensional Lie groups, whose "finite dimensional Lie subgroups" are taken to be the images of smooth finite dimensional Lie group actions.

There are several rigorous approaches to the structure of $\operatorname{Diff}(M)$. When $M$ is compact, $\operatorname{Diff}(M)$ is a Fréchet Lie group Omo74. An approach through Frölicher structures is given in [KrMi97.

In this paper we take a simpler rigorous approach: the diffeological approach. Roughly, a diffeology on a set $X$ declares which maps from $\mathbf{R}^{n}$ to $X$ are smooth, for all $n \in \mathbf{N}$. See Section 1 for relevant definitions. If $M$ is a manifold, there is a natural diffeology on $\operatorname{Diff}(M)$, with respect to which the notion "Lie subgroup of $\operatorname{Diff}(M)$ " is unambiguous: it is a subgroup that, equipped with the subset diffeology, is a manifold. The usual notion of an effective smooth action of a Lie group $G$ on a manifold $M$ is the same thing as a group monomorphism $G \rightarrow \operatorname{Diff}(M)$ that is smooth in the diffeological sense. A priori it is not obvious that in this situation the manifold structure on $G$ is induced from the ambient diffeology of $\operatorname{Diff}(M)$, i.e., that the smooth monomorphism is necessarily a diffeomorphism with its image. The purpose of this paper is to prove this fact. Thus, a Lie subgroup of $\operatorname{Diff}(M)$ really is the same thing as the image in $\operatorname{Diff}(M)$ of a smooth Lie group action on $M$. We give a formal statement in diffeological terms:

Theorem. Let $M$ be a manifold. Equip Diff( $M)$ with the functional diffeology. Then every smooth monomorphism from a Lie group to $\operatorname{Diff}(M)$ is an induction.

We now rephrase this statement in more common terms. Let $\rho: G \rightarrow \operatorname{Diff}(M)$ be an effective smooth action of a Lie group $G$ on a manifold $M$. That is, $\rho$ is a

Received by the editors November 30, 2010.

2010 Mathematics Subject Classification. Primary 58B25.

This research is partially supported by an NSERC Discovery Grant.

(c)2011 American Mathematical Society Reverts to public domain 28 years from publication 
one-to-one group homomorphism, and the map $(g, m) \mapsto \rho(g)(m)$ from $G \times M$ to $M$ is smooth. Let $D$ be an open subset of $\mathbf{R}^{k}$ for some $k$. Let $r \mapsto f_{r}$ be a map from $D$ to the image of $G$ in $\operatorname{Diff}(M)$. Let $r \mapsto g_{r}$ be the map from $D$ to $G$ such that $\rho\left(g_{r}\right)=f_{r}$ for all $r \in D$. Assume that the map $(r, m) \mapsto f_{r}(m)$, from $D \times M$ to $M$, is smooth. Then the map $r \mapsto g_{r}$, from $D$ to $G$, is smooth.

For example, this theorem immediately implies that the ordinary smooth structure of the linear group $\operatorname{GL}(n, \mathbf{R})$ is induced by the functional diffeology of $\operatorname{Diff}\left(\mathbf{R}^{n}\right)$. This fact was proved in Igl85 but not as a consequence of such a general theorem. More generally, most of the classical Lie groups (the orthogonal groups, the unitary groups, the symplectic groups, etc.) are Lie subgroups (in the diffeological sense) of groups of diffeomorphisms of vector spaces.

Sentences such as "What are, modulo conjugacy, the maximal subtori of a group of symplectomorphisms?", as considered in KKP07, have an intrinsic diffeological meaning. The above theorem shows that, fortunately, this meaning coincides with the usual interpretation of such sentences.

In Section 2, we prove the above theorem. In Section 3, we give a simple sufficient condition for which the induction is an embedding; this condition holds for the classical Lie groups that are listed above.

Throughout this paper, all manifolds, and in particular all Lie groups, are assumed to be Hausdorff, second countable, and finite dimensional.

\section{The DIFFEOLOGICAL FRAMEWORK}

We review here definitions and constructions in diffeology that we will need. More details can be found in PIZ05-10.

Let $X$ be a set. A parametrization on $X$ is a map from an open subset of $\mathbf{R}^{n}$, for some $n \in \mathbf{N}$, to $X$. A diffeology on $X$ is a set of parametrizations, whose elements are called plots, that satisfies three axioms: the covering axiom - constant maps are plots; the locality axiom - being a plot is a local condition: given $f: U \rightarrow X$, if every point in $U$ has a neighbourhood $V$ such that $\left.f\right|_{V}$ is a plot, then $f$ is a plot; and the smooth compatibility axiom - precomposition of a plot with a smooth map (in the usual sense) is a plot. A diffeological space is a set equipped with a diffeology.

Let $M$ be a manifold. There exists on $M$ a natural diffeology: the plots consist of those parametrizations that are smooth in the usual sense. On the group $\operatorname{Diff}(M)$ of diffeomorphisms of $M$ there also exists a natural diffeology, called the functional diffeology: the plots consist of those parametrizations $U \rightarrow \operatorname{Diff}(M), r \mapsto f_{r}$, for which the map $(r, m) \mapsto f_{r}(m)$ from $U \times M$ to $M$ is smooth.

The discrete diffeology on a set $X$ is the diffeology for which only the locally constant parametrizations are plots. A space with the discrete diffeology is called discrete.

A smooth map between two diffeological spaces is a map whose precomposition with every plot of the source is a plot of the target. Diffeological spaces and their smooth maps form a category. The isomorphisms of this category are called diffeomorphisms. Thus, a diffeomorphism is a bijective map that is smooth and whose inverse is smooth.

On any subset $A$ of a diffeological space $X$, there exists a natural diffeology, called the subset diffeology. Its plots are the plots of $X$ with values in $A$. If this 
diffeology is discrete, $A$ is called a discrete subspace. In particular, any countable subset of a manifold inherits the discrete diffeology, for example $\mathbf{Q} \subset \mathbf{R}$.

An induction is an injective smooth map between diffeological spaces that is a diffeomorphism onto its image, where the image is equipped with the subset diffeology. For manifolds, every induction is an immersion, and every immersion is locally an induction, but an immersion need not be an induction, even if it is injective. For example, the parametrization $t \mapsto(\sin (t), \sin (2 t))$ from $(-\pi,+\pi)$ to $\mathbf{R}^{2}$, of the lemniscate (figure eight), is an injective immersion, but it is not an induction.

A diffeology on $X$ determines a topology on $X$, called the $D$-topology, in which a subset of $X$ is open if and only if its preimage by every plot is open. Such a subset is called $D$-open. If a subset of $X$ is $\mathrm{D}$-open, then its subset topology coincides with the D-topology of its subset diffeology. The quotient $\mathbf{R} /(\mathbf{Z}+\alpha \mathbf{Z})$, for $\alpha$ irrational, is an example of a highly nontrivial 1 diffeological space whose $\mathrm{D}$-topology is trivial.

On a manifold, the D-topology coincides with the classical topology. A manifold can be redefined as a diffeological space in which every point is contained in a Dopen set that is diffeomorphic to an open subset of some $\mathbf{R}^{n}$. A map of manifolds is smooth in the usual sense if and only if it is smooth in the diffeological sense. A submanifold is just a subspace that is a manifold.

The D-topology distinguishes between inductions: an induction that is also a homeomorphism with its image, when the image is taken with the subset topology induced from the D-topology of the ambient space, is called an embedding. Not every induction is an embedding. For example, the irrational line $\mathbf{R} \rightarrow \mathbf{R}^{2} / \mathbf{Z}^{2}$ given by $x \mapsto[x, \alpha x]$ for $\alpha$ irrational is an induction but is not an embedding.

In particular, the image of the irrational line is a submanifold. This notion of "submanifold" is not quite equivalent to the notions that one finds in most textbooks. But the textbook definitions vary; they are not equivalent to each other either. The diffeological meaning of "embedded submanifold" is consistent with its meaning in most textbooks.

The quotient of a diffeological space $X$ by any equivalence relation $\mathcal{R}$ has a natural diffeology, called the quotient diffeology. Its plots are those parametrizations that can be locally lifted to plots of $X$ along the projection $\pi: X \rightarrow X / \mathcal{R}$. Thus, $f: U \rightarrow X / \mathcal{R}$ is a plot if and only if for every point $u \in U$ there exists a neighbourhood $V$ of $u$ in $U$ and a plot $\tilde{f}: V \rightarrow X$ such that $\left.f\right|_{V}=\pi \circ \tilde{f}$. A strict map $f: X \rightarrow Y$ is a smooth map that identifies the quotient $X / f$ with the image $f(X)$, as diffeological spaces [Don84.

A diffeological group $G$ is a group equipped with a diffeology such that the inversion and the multiplication are smooth. If $M$ is a manifold, then $\operatorname{Diff}(M)$, with its functional diffeology, is a diffeological group. A Lie group is the same thing as a diffeological group that is also a manifold. A Lie subgroup of $\operatorname{Diff}(M)$ is a subgroup that, with the subset diffeology, is a Lie group.

A smooth action of a diffeological group $G$ on a manifold $M$ is a smooth homomorphism $\rho: G \rightarrow \operatorname{Diff}(M)$, where $\operatorname{Diff}(M)$ is equipped with the functional diffeology. When $G$ is a Lie group, this is equivalent to the usual definition of a smooth $G$ action on $M$ : the map $(g, m) \mapsto \rho(g)(m)$ is required to be smooth. The

\footnotetext{
${ }^{1} \mathbf{R} /(\mathbf{Z}+\beta \mathbf{Z})$ is diffeomorphic to $\mathbf{R} /(\mathbf{Z}+\alpha \mathbf{Z})$ if and only if there exists $\left(\begin{array}{ll}a & b \\ c & d\end{array}\right) \in \mathrm{GL}(2, \mathbf{Z})$ such that $\beta=\frac{a \alpha+b}{c \alpha+d}$ DonIgl85.
} 
action is effective if the homomorphism $\rho$ is injective. The tautological action of $\operatorname{Diff}(M)$ on $M$ is smooth and effective.

\section{Smooth Lie Group ACtions on manifolds}

In the following paragraphs, we use the following notation. $G$ is a Lie group, $M$ is a manifold, and $\rho: G \rightarrow \operatorname{Diff}(M)$ is a smooth action of $G$ on $M$. We denote the stabilizer of $m$ in $G$ by $G_{m}$. We denote $\rho(g)(m)$ by $g \cdot m$, and we denote the $G$-orbit of $m$ by $G \cdot m$. For every point $m$ of $M$, we denote by $m_{\natural}: G \rightarrow M$ the orbit map $m_{\natural}(g):=g \cdot m$.

The orbit map $m_{\natural}$ descends to a smooth map $\bar{m}_{\natural}: G / G_{m} \rightarrow M$. Thus, $m_{\natural}=$ $\bar{m}_{\natural} \circ \pi$, where $\pi: G \rightarrow G / G_{m}$ is the projection. Moreover, because the stablizer $G_{m}$ of $m$ is a closed subgroup of $G$, this stabilizer $G_{m}$ is an embedded submanifold of $G$, the quotient $G / G_{m}$ is a manifold, and the projection $\pi: G \rightarrow G / G_{m}$ is a submersion. Moreover, $\pi$ is a (locally trivial) principal bundle with $G_{m}$ as structure group acting on $G$ by $G_{m} \times G \ni(h, g) \mapsto g h^{-1}$; see for example KobNom63.

1. The orbit map. Let $M$ be a manifold, let $G$ be a Lie group, and let $\rho: G \rightarrow$ $\operatorname{Diff}(M)$ be a smooth action of $G$ on $M$. Let $m$ be a point of $M$. Then the orbit map $m_{\natural}: G \rightarrow M$ is a strict map.

Note 1. This statement means that the map $\bar{m}_{\natural}$ is a diffeomorphism from $G / G_{m}$ to $G \cdot m$, where the former is equipped with the quotient diffeology of $G$ and the latter is equipped with the subset diffeology induced from $M$. In other words, $\bar{m}_{\natural}$ is an induction, which implies in particular that the orbit $G \cdot m$ is a submanifold (in the diffeological sense).

Note 2. The induction $\bar{m}_{\natural}: G / G_{m} \rightarrow M$ is not necessarily an embedding. For example, consider the group $G=\mathbf{R}$, the manifold $M=\mathbf{R}^{2} / \mathbf{Z}^{2} \times \mathbf{R}$, and the action $\rho(t)([x, y], \alpha)=([x+t, y+\alpha t], \alpha)$. Let $m=([x, y], \alpha)$. If $\alpha$ is rational, then $m_{\natural}$ is an embedding, and if $\alpha$ is irrational, then $m_{\natural}$ is an induction but not an embedding.

Proof. Because the quotient map $\pi: G \rightarrow G / G_{m}$ admits smooth local sections, the map $\bar{m}_{\natural}: G / G_{m} \rightarrow M$ is a smooth injection. Let $\sigma$ be a smooth section of $\pi$, let $\mathcal{O}$ be its image in $G$, and assume that $\mathbf{1}_{G} \in \mathcal{O}$. Then $\mathcal{O}$ is transverse to $G_{m}$ at the identity $\mathbf{1}_{G}$.

The differential of the orbit map $m_{\natural}: g \mapsto g \cdot m$, computed at the identity $\mathbf{1}_{G}$, is the map $\xi \mapsto \xi_{M}(m)$, where $\xi$ is an element of the Lie algebra $\mathcal{G}$ of $G$ and $\xi_{M}$ denotes the fundamental vector field on $M$ associated to $\xi$. The kernel of this map is equal to the Lie algebra $\mathcal{G}_{m}$ of $G_{m}$ :

$$
\mathcal{G}_{m}=T_{\mathbf{1}_{G}} G_{m}=\left\{\xi \in \mathcal{G} \mid \xi_{M}(m)=0\right\} .
$$

The image of this map is the subspace $\mathcal{G} \cdot m=\left\{\xi_{M}(m) \mid \xi \in \mathcal{G}\right\}$ of $T_{m} M$. Let $D$ be a disc in $M$ through $m$ that is transverse at $m$ to this subspace.

Consider the map

$$
\psi: \mathcal{O} \times D \rightarrow M, \quad(a, x) \mapsto a \cdot x .
$$

By the implicit function theorem, after possibly shrinking $\mathcal{O}$ and $D$, the image $U_{m}:=\psi(\mathcal{O} \times D)$ is open in $M$, and $\psi$ is a diffeomorphism of $\mathcal{O} \times D$ with $U_{m}$.

For every $x \in D$, consider the set

$$
A_{x}:=\{g \in G \mid g \cdot m \in \psi(\mathcal{O} \times\{x\})\} .
$$


Note that $A_{x}$ is nonempty if and only if $x \in G \cdot m \cap D$.

Let us now show that

$$
G \cdot m \cap D \text { is countable. }
$$

For this, we will first show that

$(\diamond)$ for every $x \in D$, the set $A_{x}$ is an open subset of $G$;

(Q) for every $x, y \in D$, if $x \neq y$, then $A_{x} \cap A_{y}=\varnothing$.

Let $g \in G$. Suppose that $g \cdot m=a \cdot x$ for $a \in \mathcal{O}$. The subset $\mathcal{O} \cdot G_{x} \cdot a^{-1} \cdot g$ of $G$ is a neighbourhood of $g$ in $G$, and for every $g^{\prime}$ in this subset, $g^{\prime} \cdot m \in \psi(\mathcal{O} \times\{x\})$. This shows $(\diamond)$.

Now suppose that $g$ is in both $A_{x}$ and $A_{y}$. Then both $x$ and $y$ are equal to $\operatorname{pr}_{D} \circ \psi^{-1}(g \cdot m)$, where $\psi^{-1}: U_{m} \rightarrow \mathcal{O} \times D$ is the inverse of $\psi$ and where $\operatorname{pr}_{D}: \mathcal{O} \times$ $D \rightarrow D$ is the projection to the second coordinate. So $x$ and $y$ are equal to each other. This shows $(\mathcal{Q})$.

Thus, $\left\{A_{x}\right\}_{x \in D}$ is a family of disjoint open subsets of $G$. Because $G$ is second countable, at most countably many elements of this family are nonempty. But $A_{x}$ is nonempty exactly if $x \in G \cdot m \cap D$. This shows (\$o).

Let us now check that the map $\bar{m}_{\natural}: G / G_{m} \rightarrow M$ is an induction (diffeomorphism with its image). Fix an arbitrary parametrization $\bar{\gamma}: V \rightarrow G / G_{m}$, and assume that the composition $\bar{m}_{\natural} \circ \bar{\gamma}$ is smooth. We need to show that $\bar{\gamma}$ itself is smooth.

Let $r_{0}$ be a point in $V$ and let $g \in G$ be such that $\bar{\gamma}\left(r_{0}\right)$ is equal to the coset $g G_{m}$ in $G / G_{m}$. We need to find a neighbourhood of $r_{0}$ in $V$ in which $\bar{\gamma}$ is smooth. Shrinking $V$, precomposing $\bar{\gamma}$ by translation by $r_{0}$, and composing $\bar{\gamma}$ by left multiplication by the element $g^{-1}$ of $G$, we reduce the problem to the following special case.

We assume that $V$ is a small open ball centred at the origin 0 , that $\bar{\gamma}(0)=\pi\left(\mathbf{1}_{G}\right)$, and that $\bar{\gamma}(V) \subset \pi(\mathcal{O})$. The map $\gamma:=\sigma \circ \bar{\gamma}: V \rightarrow \mathcal{O}$ is then well defined, and it is a lift of $\bar{\gamma}$, that is, $\pi \circ \gamma=\bar{\gamma}$. The map $v \mapsto \gamma(v) \cdot m$, being equal to the composition $\bar{m}_{\natural} \circ \bar{\gamma}$, is smooth by assumption; also, it takes values in the subset $U_{m}(=\psi(\mathcal{O} \times D))$ of $M$.

By the definition of $\psi$, we can write

$$
\psi^{-1}(\gamma(v) \cdot m)=(\alpha(v), \mu(v)),
$$

where $\alpha$ is a parametrization of $G$ that takes values in $\mathcal{O}$, where $\mu$ is a parametrization of $M$ that takes values in $D$, and where

$$
\alpha(v) \cdot \mu(v)=\gamma(v) \cdot m
$$

for all $v \in V$. Rewriting the last equality as $\mu(v)=\left(\alpha(v)^{-1} \gamma(v)\right) \cdot m$, we see that $\mu$ also takes values in $G \cdot m$. So $\mu$ takes values in the set $G \cdot m \cap D$, which is countable by $(\boldsymbol{\beta})$.

Because $v \mapsto \psi^{-1}(\gamma(v) \cdot m)$, being the composition of two smooth maps, is smooth, the parametrizations $\alpha$ and $\mu$ are also smooth. Being a smooth map on an open ball that takes values in a countable set, the map $\mu$ is constant. So $\mu(v)=m$ for all $v$, and $\alpha(v) \cdot m=\gamma(v) \cdot m$ for all $v$. This, in turn, implies that $\pi(\alpha(v))=\pi(\gamma(v))$ for all $v$. Because $\alpha(v)$ and $\gamma(v)$ both belong to $\mathcal{O}$, composing with $\sigma$ gives $\gamma(v)=\alpha(v)$. But we already know that $\alpha$ is smooth. So $\gamma$ is also smooth, and, hence, so is $\bar{\gamma}$. 
2. When $G$ is Countable. Let $M$ be a manifold, $G$ a countable discrete group, and $\rho: G \rightarrow \operatorname{Diff}(M)$ a smooth action. Then the image of $\rho$ is a discrete subset of $\operatorname{Diff}(M)$. In particular, if the action is effective then $\rho$ is an induction.

Note 1. Because we assume manifolds to be second countable, a countable discrete group is the same thing as a zero dimensional Lie group.

Note 2. The image of a discrete diffeological group into Diff $(M)$ by a smooth monomorphism has no reason to be discrete a priori. For example, a Lie group $G$ injects into $\operatorname{Diff}(G)$ by left multiplication. Let $G_{\circ}$ denote the set $G$ equipped with the discrete diffeology; then the injection $G_{\circ} \rightarrow \operatorname{Diff}(G)$ is still smooth, but it is not an induction if $\operatorname{dim} G>0$. We rule out this example by assuming that $G$ is countable.

Proof. Let $r \mapsto f_{r}$ be a smooth parametrization in $\operatorname{Diff}(M)$ with values in $\rho(G)$, and let $r_{0}$ be a point in the domain of this parametrization. Fix $m \in M$. Because $G$ is countable, the orbit $G \cdot m$ is countable. But a countable subspace of a manifold is diffeologically discrete. So the smooth parametrization $\varphi: r \mapsto f_{r}(m)$, which takes its values in the countable subspace $G \cdot m$ of $M$, is locally constant. But locally constant means constant on the connected components of the domain of the parametrization [PIZ05-10, chap. II]. Let $B$ be an open ball in the domain of $\varphi$ that is centred at $r_{0}$. Then $f_{r}(m)=f_{r_{0}}(m)$ for all $r$ in $B$. Repeating this argument for all $m$ in $M$, we deduce that $f_{r}=f_{r_{0}}$ for all $r$ in $B$. Thus, the parametrization $r \mapsto f_{r}$ is locally constant. Therefore, the image of $G$ by $\rho$ is discrete. It follows immediately that if the action is effective then $\rho$ is an induction.

3. The CASe of A Discrete Stabilizer. Let $M$ be a manifold, let $G$ be a Lie group, and let $\rho: G \rightarrow \operatorname{Diff}(M)$ be an effective smooth action of $G$ on $M$. Suppose that there exists a point $m$ in $M$ such that the stabilizer $G_{m}$ is discrete. Then $\rho$ is an induction.

Proof. Let $r \mapsto f_{r}$ be a parametrization of $\operatorname{Diff}(M)$ that takes its values in $\rho(G)$. Let $r \mapsto g_{r}$ be the parametrization of $G$ such that $\rho\left(g_{r}\right)=f_{r}$ for all $r$. Let $r_{0}$ be a point in the domain of these parametrizations. We would like to show that $r \mapsto g_{r}$ is smooth near $r_{0}$.

Let $m$ be a point of $M$ whose stabilizer $G_{m}$ is discrete. The parametrization $r \mapsto f_{r}(m)$ of $M$ takes its values in the orbit $G \cdot m$. Since $\bar{m}_{\natural}: G / G_{m} \rightarrow M$ is an induction (Paragraph 1), and by the definition of the quotient diffeology on $G / G_{m}$, there exists a neighbourhood $B$ of $r_{0}$ and a smooth map $r \mapsto g_{r}^{\prime}$ from $B$ to $G$ such that $f_{r}(m)=\rho\left(g_{r}^{\prime}\right)(m)$ for all $r \in B$. The map $r \mapsto \rho\left(g_{r}^{\prime}\right)^{-1} \circ f_{r}$ is a plot of $\operatorname{Diff}(M)$ that takes its value in $\rho\left(G_{m}\right)$. But since $G_{m}$ is assumed to be discrete, $\rho\left(G_{m}\right)$ is also discrete (Paragraph 2). So, $r \mapsto \rho\left(g_{r}^{\prime}\right)^{-1} \circ f_{r}$ is locally constant. Therefore, after possibly shrinking the neighbourhood $B$ of $r_{0}$, there exists a constant element $\kappa \in G_{m}$ such that $\rho\left(g_{r}^{\prime}\right)^{-1} \circ f_{r}=\rho(\kappa)$, that is, $f_{r}=\rho\left(g_{r}^{\prime} \kappa\right)$, for all $r \in B$. But also $f_{r}=\rho\left(g_{r}\right)$. Because the action is effective, we deduce that $g_{r}=g_{r}^{\prime} \kappa$ for all $r \in B$. Because $r \mapsto g_{r}^{\prime}$ is smooth and $\kappa$ is constant, this implies that $r \mapsto g_{r}$ is smooth.

4. IntERSECTING VECTOR SUBSPACES. Let $E$ be a finite dimensional vector space. Let $\left\{E_{i}\right\}_{i \in \mathcal{I}}$ be a family of subspaces of $E$. If $\bigcap_{i \in \mathcal{I}} E_{i}=\{0\}$, then there exists a finite set of indices $(i, j, \ldots, k) \subset \mathcal{I}$ such that $E_{i} \cap E_{j} \cap \cdots \cap E_{k}=\{0\}$. 
Proof. We prove the result by induction on the dimension of $E$. If $E=\{0\}$, the result is trivial: we can take the finite set of indices to be the empty set. Suppose that the result is true for vector spaces of dimension smaller than that of $E$. Because $\bigcap_{i \in \mathcal{I}} E_{i}=\{0\}$, there exists at least one vector subspace $E_{i}$ such that $E_{i} \neq E$, that is, $\operatorname{dim}\left(E_{i}\right)<\operatorname{dim}(E)$. Let us define $\mathcal{I}^{\prime}:=\mathcal{I} \backslash\{i\}, E^{\prime}:=E_{i}$, and $E_{j}^{\prime}:=$ $E^{\prime} \cap E_{j}$ for all $j \in \mathcal{I}^{\prime}$. We have $\bigcap_{j \in \mathcal{I}} E_{j}=\bigcap_{j \in \mathcal{I}^{\prime}} E_{j}^{\prime}$. Thus, $\left\{E_{j}^{\prime}\right\}_{j \in \mathcal{I}^{\prime}}$ is a family of vector subspaces of $E^{\prime}\left(=E_{i}\right)$ with $\bigcap_{j \in \mathcal{I}^{\prime}} E_{j}^{\prime}=\{0\}$. By the induction hypothesis, there is a finite set of indices $\{j, \ldots, k\} \subset \mathcal{I}^{\prime}$ such that $E_{j}^{\prime} \cap \ldots \cap E_{k}^{\prime}=\{0\}$. The union of this set with the index $\{i\}$ is a finite set of indices $\{i, j, \ldots, k\} \subset \mathcal{I}$ such that $E_{i} \cap E_{j} \cap \cdots \cap E_{k}=\{0\}$.

5. Obtaining a Discrete intersection of Stabilizers. Let $M$ be a manifold, $G$ a Lie group, and $\rho: G \rightarrow \operatorname{Diff}(M)$ an effective smooth action. Then there exists a finite set of points $\left\{m_{1}, \ldots, m_{N}\right\} \subset M$ such that the intersection of their stabilizers, $G_{m_{1}} \cap \cdots \cap G_{m_{N}}$, is discrete.

Proof. Denote by $\mathcal{G}$ the Lie algebra of $G$, and, for every point $m$ of $M$, denote by $\mathcal{G}_{m}$ the Lie algebra of the stabilizer $G_{m}$ of $m$. For any smooth parametrization $P: U \rightarrow G$, if $P$ takes values in $G_{m}$, then, for every $r \in U$, the composition of the differential $d P_{r}: T_{r} U \rightarrow T_{P(r)} \mathcal{G}$ with the left translation by $P(r)^{-1}$ takes values in the subspace $\mathcal{G}_{m}$ of $\mathcal{G}$.

Because the action is effective, $\bigcap_{m \in M} G_{m}=\{1\}$. Recall that $\mathcal{G}_{m}=\{\xi \in$ $\mathcal{G} \mid \exp (t \xi) \in G_{m}$ for all $\left.t \in \mathbf{R}\right\}$. It follow that $\bigcap_{m \in M} \mathcal{G}_{m}=\{0\}$. According to Paragraph 4, there exists a finite set of points, say, $\left\{m_{1}, \ldots, m_{N}\right\} \subset M$, such that $\mathcal{G}_{m_{1}} \cap \cdots \cap \mathcal{G}_{m_{N}}=\{0\}$.

Let $P: U \rightarrow G$ be a smooth parametrization that takes values in $G_{m_{1}} \cap \cdots \cap G_{m_{N}}$. For every $r \in U$, the composition of the differential $d P_{r}: T_{r} U \rightarrow T_{P(r)} G$ with the left translation by $P(r)^{-1}$ takes values in $\mathcal{G}_{m_{1}} \cap \cdots \cap \mathcal{G}_{m_{N}}$. So the differential $d P_{r}$ is zero for every $r \in U$, and so the map $P$ is locally constant. Therefore, $G_{m_{1}} \cap \cdots \cap G_{m_{N}}$ is discrete.

6. Smooth Lie group actions. Let $M$ be a manifold, $G$ a Lie group, and $\rho: G \rightarrow \operatorname{Diff}(M)$ an effective smooth action. Then $\rho$ is an induction. That is, $\rho$ is a diffeomorphism onto its image, when the image is equipped with the subset diffeology.

Note 3. Let $M$ be a manifold, $G$ a Lie group, and $\rho: G \rightarrow \operatorname{Diff}(M)$ a smooth action that is not necessarily effective. Let $K=\operatorname{ker} \rho$. Applying Claim [6 to the quotient $G / K$, we deduce that $\rho$ induces a diffeomorphism from $G / K$ to its image in $\operatorname{Diff}(M)$; in other words, $\rho$ is strict.

Proof. By Paragraph 5 , there exists a natural number $N$ and a point $\mu=\left(m_{1}, \ldots\right.$, $\left.m_{N}\right)$ in $M^{N}$ whose stabilizer $G_{\mu}$ under the diagonal action of $G$ on $M^{N}$ is discrete. Write this diagonal action as $\rho_{N}: G \rightarrow \operatorname{Diff}\left(M^{N}\right)$, that is, $\rho_{N}(g)\left(m_{1}, \ldots, m_{N}\right)=$ $\left(g \cdot m_{1}, \ldots, g \cdot m_{N}\right)$. By Paragraph 3 , the map $\rho_{N}: G \rightarrow \operatorname{Diff}\left(M^{N}\right)$ is an induction. Now, let $r \mapsto f_{r}$ be a plot of $\operatorname{Diff}(M)$ that takes its values in $\rho(G)$, and, for every $r$, let $g_{r}$ be the element of $G$ such that $f_{r}=\rho\left(g_{r}\right)$. Let $f_{N, r}\left(m_{1}, \ldots, m_{N}\right):=$ $\left(f_{r}\left(m_{1}\right), \ldots, f_{r}\left(m_{N}\right)\right)$. Then $r \mapsto f_{N, r}$ is a plot of $\operatorname{Diff}\left(M^{N}\right)$ that takes its values in $\rho_{N}(G)$, and $f_{N, r}=\rho_{N}\left(g_{r}\right)$ for all $r$. Because $\rho_{N}$ is an induction, it follows that $r \mapsto g_{r}$ is smooth. Thus, $\rho$ is an induction. 


\section{A SIMPLE CASE OF AN EMBEDDiNG}

We have proved the theorem that we stated in the introduction: an effective smooth Lie group action $\rho: G \rightarrow \operatorname{Diff}(M)$ is an induction.

It is now natural to ask when is such a $\rho$ an embedding, that is, a homeomorphism of $G$ with its image, when the image is equipped with the subset topology of the D-topology of Diff $(M)$. In other words, how do these two topologies compare? Paragraph 7 gives a sufficient condition. Applied to $\mathrm{GL}(n, \mathbf{R})$ acting naturally on $\mathbf{R}^{n}$, this condition shows again that $\operatorname{GL}(n, \mathbf{R})$ is embedded in $\operatorname{Diff}\left(\mathbf{R}^{n}\right)$ Igl85.

7. An ORBIT MAP EMBEDDING CONDITION. Let $M$ be a manifold, $G$ a Lie group, and $\rho: G \rightarrow \operatorname{Diff}(M)$ an effective smooth action. Suppose that there exists a natural number $k$ and a point $\mu=\left(m_{1}, \ldots, m_{k}\right)$ in $M^{k}$ such that the orbit map $\mu_{\natural}: G \rightarrow M^{k}$ for the diagonal action is an embedding. Then $\rho: G \rightarrow \operatorname{Diff}(M)$ is an embedding.

Note 1. Considering the $n$ standard basis vectors of $\mathbf{R}^{n}$, we conclude that $\mathrm{GL}(n, \mathbf{R})$ is embedded in $\operatorname{Diff}\left(\mathbf{R}^{n}\right)$. The same applies to classical Lie groups such as the orthogonal groups, the unitary groups, the symplectic groups, etc.

Note 2. By our main theorem, the irrational flow $R \rightarrow \operatorname{Diff}\left(T^{2}\right)$, given by $\rho(t)([x, y])=[x+t, y+\alpha t]$ with $\alpha$ irrational, is an induction, that is, a diffeomorphism with its image in $\operatorname{Diff}\left(T^{2}\right)$, but it is not an embedding. Indeed, this flow splits as a composition $R \rightarrow T^{2} \rightarrow \operatorname{Diff}\left(T^{2}\right)$, where the second arrow is an embedding (every diffeological group is embedded through left translation in its group of diffeomorphisms [PIZ05-10, chap. VII]), but the first arrow is not (cf. Note 2 of Paragraph 1).

Proof. Let $B \subset G$ be an open set, and let

$$
U=\mu_{\natural}(B)=\left\{\left(g \cdot m_{1}, \ldots, g \cdot m_{k}\right) \in M^{k} \mid g \in B\right\} .
$$

Since, by assumption, $\mu_{\natural}$ is an embedding, it is a fortiori an induction, and $U$ is open in $G \cdot \mu$ with respect to the subset topology. So there exists an open subset $V \subset M^{k}$ such that $U=V \cap G \cdot \mu$. Let us then introduce the set

$$
\Omega=\left\{f \in \operatorname{Diff}(M) \mid\left(f\left(m_{1}\right), \ldots, f\left(m_{k}\right)\right) \in V\right\} .
$$

Let us now check that $\Omega$ is open for the D-topology of the functional diffeology and that $\rho(B)=\rho(G) \cap \Omega$. This will prove the claim.

Let $P: \mathcal{O} \rightarrow \operatorname{Diff}(M)$ be a plot. The preimage $P^{-1}(\Omega)$ consists of those $r \in \mathcal{O}$ such that

$$
\left(P(r)\left(m_{1}\right), \ldots, P(r)\left(m_{k}\right)\right) \in V .
$$

That is, $P^{-1}(\Omega)=\left(\mu_{\natural} \circ P\right)^{-1}(V)$. But $\mu_{\natural} \circ P$ is smooth, hence D-continuous, and $V$ is open. Thus, $P^{-1}(\Omega)$ is open. Because this is true for every plot $P: \mathcal{O} \rightarrow \operatorname{Diff}(M)$, we deduce that $\Omega$ is D-open.

Let us now check that $\rho(B)=\rho(G) \cap \Omega$. Let $g^{\prime} \in G$. By the definition of $\Omega$, the diffeomorphism $\rho\left(g^{\prime}\right)$ belongs to $\Omega$ if and only if the $k$-tuple $\left(g^{\prime} \cdot m_{1}, \ldots, g^{\prime} \cdot m_{k}\right)$ is in $V$. But this $k$-tuple is automatically in $G \cdot \mu$. So $\rho\left(g^{\prime}\right)$ belongs to $\Omega$ if and only if $\left(g^{\prime} \cdot m_{1}, \ldots, g^{\prime} \cdot m_{k}\right)$ belongs to the intersection $V \cap G \cdot \mu$, which, in turn, is equal to $U$, which is $\mu_{\natural}(B)$. Thus, $\rho\left(g^{\prime}\right)$ belongs to $\Omega$ if and only if there exists $g \in B$ such that $\left(g^{\prime} \cdot m_{1}, \ldots, g^{\prime} \cdot m_{k}\right)=\left(g \cdot m_{1}, \ldots, g \cdot m_{k}\right)$. This last condition holds exactly if $g^{-1} g^{\prime} \in G_{\mu}$. Because $G_{\mu}$ is trivial, $\rho\left(g^{\prime}\right)$ belongs to $\Omega$ if and only if $g^{\prime}=g \in B$. 
Therefore, $\Omega$ is D-open in $\operatorname{Diff}(M)$, and $\rho(B)=\rho(G) \cap \Omega$. So $\rho(B)$ is open with respect to the subset topology on $\rho(G)$ that is induced from the D-topology in $\operatorname{Diff}(M)$. The proof is complete.

\section{REFERENCES}

[Don84] Paul Donato, Revêtement et groupe fondamental des espaces différentiels homogènes. Thèse de doctorat d'état, Université de Provence, Marseille, 1984.

[DonIgl85] Paul Donato and Patrick Iglesias. Exemple de groupes difféologiques: flots irrationnels sur le tore. Compte Rendu de l'Académie des Sciences, 301(4), Paris, 1985. MR799609 (86j:58077)

[Igl85] Patrick Iglesias, Fibrés difféologiques et homotopie. Thèse de doctorat d'état, Université de Provence, Marseille, 1985.

[KobNom63] S. Kobayashi and K. Nomizu. Foundations of differential geometry. Interscience Publishers, 1963. MR0152974 (27:2945)

[KKP07] Y. Karshon, L. Kessler, and M. Pinsonnault, A compact symplectic four-manifold admits only finitely many inequivalent toric actions. J. Sympl. Geom. 5 (2007), no. 2, 139-166. MR2377250(2008m:53199)

[KrMi97] A. Kriegl and P. Michor, The convenient setting of global analysis. Amer. Math. Soc., Providence, RI, 1997. MR1471480 (98i:58015)

[Omo74] Hideki Omori, Infinite Dimensional Lie Transformation Groups. Lecture Notes in Mathematics 427. Springer-Verlag, Berlin-New York, 1974. MR0431262 (55:4263)

[PIZ05-10] Patrick Iglesias-Zemmour, Diffeology. Eprint, 2005-2010. http://math.huji. ac.il/ piz/diffeology/

Laboratoire d'Analyse, Topologie et Probabilités, CNRS, Marseille, France - and The Hebrew University of Jerusalem, Israel

E-mail address: piz@math.huji.ac.il

Department of Mathematics, The University of Toronto, 40 St. George Street, Toronto, Ontario M5S 2E4, Canada

E-mail address: karshon@math.toronto.edu 